\title{
Magnetoenzephalografie (MEG)
}

\author{
Johannes Wöhrle
}

Die Magnetoenzephalografie (MEG) ist die Messung der mit der hirnelektrischen Aktivität einhergehenden Magnetfeldveränderungen. Sie ist keine klinische Routinemethode, sondern stellt vielmehr ein interessantes Forschungsinstrument dar.

Die technisch sehr aufwendige und kostenintensive Magnetoenzephalografie beruht auf dem Prinzip der primären Messung der Magnetfelder, welche durch die elektrische Aktivität von Neuronenpopulationen des Kortex hervorgerufen werden. Diese magnetischen Felder sind sehr schwach, daher ist eine ganz besondere Abschirmung des Gerätes und seiner Umgebung vom Erdmagnetfeld und anderen elektromagnetischen Feldquellen erforderlich. Die Magnetfelder werden im Gegensatz zu der EEG-Aktivität kaum durch den über dem Kortex liegenden Liquor oder die Schädeldecke abgeschwächt, sodass eine deutlich höhere räumliche Auflösung resultiert. Noch wichtiger ist aber die zeitliche Auflösung, die im Millisekundenbereich liegt. Bei Anwendung von speziellen mathematischen Modellen können zur Dipolquellenlokalisation eher tangential zur Kortexoberfläche angeordnete Dipole bestimmt werden, die wahrscheinlich der Realität am nächsten kommen, im Gegensatz zu den aus der EEG-Aktivität errechneten eher radiär angeordneten Dipolen. Zudem lassen sich neuronale
Vorgänge in der Tiefe des Gehirns untersuchen. Die Methode wird idealerweise durch die Magnetresonanztomografie ergänzt. In der klinischen Anwendung kann die Methode besonders der Lokalisation von epileptiformer Aktivität dienen, ein wissenschaftlicher Anwendungsbereich ist beispielsweise die Quellenlokalisation von evozierten Potenzialen (Zschocke und Hansen 2012; Schomer und da Silva 2011).

\section{Facharztfragen}

1. Welche Indikationen sehen Sie in der klinischen Anwendung für die Magnetoenzephalografie?

\section{Literatur}

Schomer DL, da Silva FHL (Hrsg) (2011) Niedermeyer's electroencephalography. Basic principles, clinical applications, and related fields, 6. Aufl. Wolters Kluwer/Lippincott Williams \& Wilkins, Philadelphia

Zschocke S, Hansen HC (2012) Klinische Elektroenzephalographie, 3. Aufl. Springer, Berlin/Heidelberg/New York/Tokio
J. Wöhrle $(\bowtie)$

Neurologische Klinik, Katholisches Klinikum Koblenz Montabaur, Brüderhaus Koblenz, Koblenz, Deutschland

E-Mail: neurologie@kk-km.de; j.woehrle@kk-km.de

(C) Springer-Verlag GmbH Deutschland 2017

P. Berlit (Hrsg.), Klinische Neurologie, Springer Reference Medizin, DOI 10.1007/978-3-662-44768-0_10-1 